\title{
Enhanced anticancer effect of ABT-737 in combination with naringenin on gastric cancer cells
}

\author{
HAIYANG ZHANG ${ }^{1}$, XIA ZHONG ${ }^{1}$, XIAO ZHANG ${ }^{2}$, DEYA SHANG ${ }^{1}$, YI ZHOU ${ }^{1}$ and CHUNQING ZHANG ${ }^{3}$ \\ ${ }^{1}$ Department of Emergency, Shandong Provincial Hospital Affiliated to Shandong University, Jinan, Shandong 250021; \\ ${ }^{2}$ Shandong Center for Disease Control and Prevention, Jinan, Shandong 250014; ${ }^{3}$ Division of Hepatogastroenterology, \\ Department of Internal Medicine, Shandong Provincial Hospital Affiliated to Shandong University, \\ Jinan, Shandong 250021, P.R. China
}

Received January 18, 2015; Accepted October 22, 2015

DOI: 10.3892/etm.2015.2912

\begin{abstract}
Gastric cancer is the second leading cause of cancer-associated mortality and is a frequently occurring cancer worldwide. Multiple drug resistance of gastric cancer cells leads to the poor prognosis. In addition, overexpression of anti-apoptotic protein $\mathrm{B}$-cell lymphoma $(\mathrm{Bcl})-2$ have been demonstrated in various cancer cells and is closely associated with drug resistance and poor prognosis. Naringenin is a flavonoid that has antimutagenic and anticarcinogenic activities in numerous cancer types. In the present study, naringenin and a Bcl-2 inhibitor, ABT-737, were used to investigate their combinative anticancer effect in the SGC7901 gastric cancer cell line. The results revealed that naringenin and ABT-737 were able to inhibit SGC7901 cell growth and colony formation, alone or in combination. Furthermore, the combination of these drugs was found to further increase the cleavage of caspase-3 and poly ADP-ribose polymerase. Naringenin and ABT-737 also decreased Akt activation and increased p53 expression, suggesting the involvement of these pathways in the inhibition of gastric cell growth.
\end{abstract}

\section{Introduction}

Gastric cancer is the second leading cause of cancer-associated mortality worldwide (1). As one of the most frequently occurring cancer types (2-3\% of all cases of cancer) (2), and with a low survival rate (29\% at 5 years) (3), gastric cancer is mainly treated with surgery and combination chemotherapy regimens, which have improved the overall survival rates (4). However, poor prognosis caused by multiple drug resistance remains the major problem in cancer treatment. Improved or novel

Correspondence to: Dr Chunqing Zhang, Division of Hepatogastroenterology, Department of Internal Medicine, Shandong Provincial Hospital Affiliated to Shandong University, 324 Jingwu Weiqi Road, Jinan, Shandong 250021, P.R. China

E-mail: chunqingzhang@126.com

Key words: ABT-737, naringenin, gastric cancer, anticancer combination chemotherapy strategies must be developed in order to overcome drug resistance and improve the prognosis of gastric cancer patients.

Flavonoids, which are mainly extracted from plants or fruit, have multiple biological activities and have been widely studied in cancer therapy $(5,6)$. Naringenin is a flavonoid that is extracted from citrus fruit and possesses antimutagenic and anticarcinogenic activities $(7,8)$. Recent studies have shown that naringenin is able to induce cancer growth inhibition, migration or cell cycle arrest in various cancer cells, including epidermoid carcinoma, human hepatocellular carcinoma, bladder cancer and breast cancer cells (8-11). However, to the best of our knowledge, the anticancer activity of naringenin in gastric cancer cells has not been studied.

Overexpression of the anti-apoptotic protein, B-cell lymphoma $(\mathrm{Bcl})-2$, has been commonly observed in various cancer cells and is associated with drug resistance and poor prognosis (12). ABT-737, a small molecule mimicking BH3-only protein, is an inhibitor of $\mathrm{Bcl}-2$ and its anticancer activity has been studied in lung cancer (13), acute myeloid leukemia (14), multiple myeloma (15) and lymphoma (16). However, the effect of ABT-737 on gastric cancer cells has not been extensively investigated (17).

In the present study, the combinative effect of naringenin and ABT-737 on gastric cancer cells was investigated. Cancer cell growth and colony formation were examined in the gastric cancer cell line, SGC7901. In addition, the potential underlying mechanism of action of naringenin and ABT-737 was analyzed.

\section{Materials and methods}

Cell culture andreagents. The SGC7901 human gastric cancer cell line (American Type Culture Collection, Manassas, VA, USA) was maintained in RPMI-1640 medium (Invitrogen; Thermo Fisher Scientific, Inc., Waltham, MA, USA), supplemented with $10 \%$ fetal bovine serum, glutamine and penicillin and streptomycin antibiotics (Invitrogen; Thermo Fisher Scientific, Inc.) at $37^{\circ} \mathrm{C}$ in $5 \% \mathrm{CO}_{2}$. Naringenin and ABT-737 were obtained from Sigma-Aldrich (St. Louis, MO, USA) and Selleck Chemicals (Houston, TX, USA), respectively. 
Antibodies and reagents. Mouse anti-human poly ADP-ribose polymerase (PARP) polyclonal antibody (pAb; 1:1,000; 9542), rabbit anti-human protein kinase B (Akt) pAb (1:1,000; 9272), and rabbit anti-human phospho-Akt (p-Akt) pAb $(1: 1,000 ; 9271)$ were purchased from Cell Signaling Technology, Inc. (Danvers, MA, USA). Rabbit anti-human caspase-3 pAb (1:1,000; AC030), mouse anti-human p53 monoclonal antibody (mAb; 1:1,000; AP062) and mouse anti-human glyceraldehyde-3-phosphate dehydrogenase (GAPDH) mAb (1:800; AG019), as well as horseradish peroxidase (HRP)-conjugated goat anti-rabbit $(1: 1,000 ; A 0239)$ and goat anti-mouse $(1: 1,000 ; A 0216) \operatorname{IgG}$ secondary antibodies, were purchased from Beyotime Institute of Biotechnology (Shanghai, China).

Cell viability assay. The effect of naringenin or ABT-737 on SGC7901 cell growth was measured using the cell counting kit-8 assay (CCK-8; Beyotime Institute of Biotechnology). Briefly, 2,000 SGC7901 cells cultured in a 96-well plate were treated with naringenin $(20,40,80$, or $160 \mu \mathrm{M})$, ABT-737 $(1,5$, 10 , or $15 \mu \mathrm{M})$ or a combination of the two $(40 \mu \mathrm{M}$ naringenin and $5 \mu \mathrm{M} \mathrm{ABT-737)}$ for $48 \mathrm{~h}$. Subsequent to the treatment, $10 \mu$ l WST-8 solution (Beyotime Institute of Biotechnology) was added and the cells were incubated for a further $2 \mathrm{~h}$. The number of viable cells was measured with a microplate reader (Bio-Rad Laboratories, Inc., Hercules, CA, USA) at a test wavelength of $450 \mathrm{~nm}$. Cell viability was normalized against control cells treated with vehicle (dimethyl sulfoxide) and GraphPad Prism 5 software (GraphPad Software, Inc., La Jolla, CA, USA) was used to produce histograms and analyze the data. All cell viability assays were performed in triplicate and repeated in three independent experiments.

Colony formation assay. For the colony formation assay, 500 SGC7901 cells were seeded in a 6-well plate and incubated with $40 \mu \mathrm{M}$ naringenin, $5 \mu \mathrm{M}$ ABT-737 or a combination of the two for 6 days. The cell colonies were fixed with methanol for $15 \mathrm{~min}$ at room temperature and then stained with crystal violet staining solution (Beyotime Institute of Biotechnology) for $30 \mathrm{~min}$. Colonies with a diameter $>0.2 \mathrm{~mm}$ were counted and the results were presented as a histogram. Colony formation experiments were performed in triplicate and repeated in three independent experiments.

Western blot analysis. SGC7901 cells were treated with $40 \mu \mathrm{M}$ naringenin and/or $5 \mu \mathrm{M}$ ABT-737 for $48 \mathrm{~h}$, after which the cells were suspended in cell lysis buffer (Beyotime Institute of Biotechnology) supplemented with a protease inhibitor cocktail, in order to extract whole cell lysates. The lysates were resolved by $12.5 \%$ sodium dodecyl sulfate-polyacrylamide gel electrophoresis and then transferred to polyvinylidene difluoride membranes (Beyotime Institute of Biotechnology). Subsequently, the membranes were blocked with 5\% non-fat milk. Next, they were incubated with the appropriate primary antibody subsequent to washing with phosphate-buffered saline containing $1 \%$ Tween 20 , followed by exposure to the HRP-conjugated anti-rabbit IgG secondary antibody. The bands were visualized by the addition of an enhanced chemiluminescence reagent (Beyotime Institute of Biotechnology) to the membranes. Images were captured using X-ray films (Kodak, Rochester, NY, USA) in a dark room.
$\mathbf{A}$

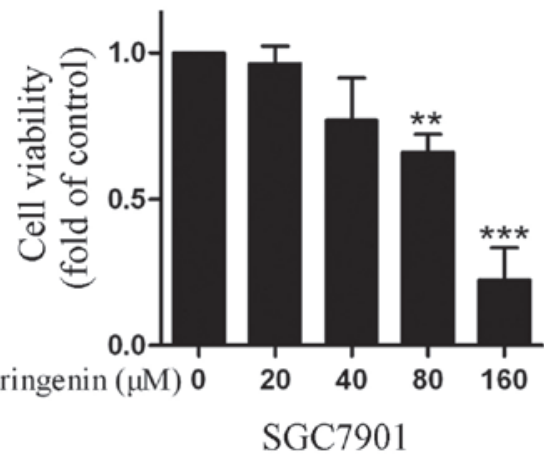

B

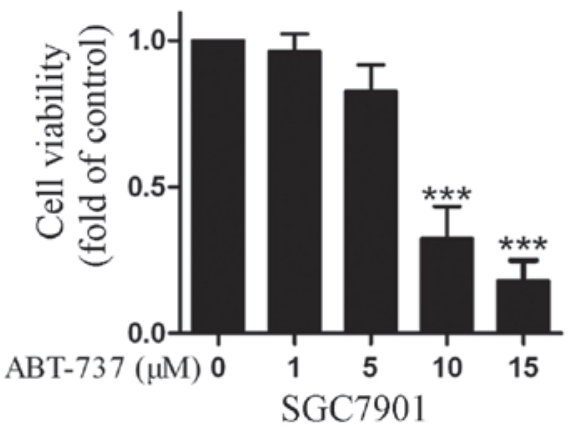

C

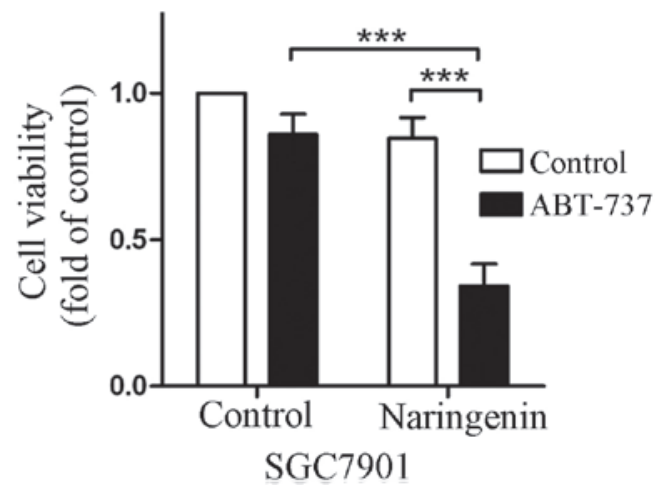

Figure 1. Naringenin and ABT-737 inhibited SGC7901 cell growth. SGC7901 cells were treated with various concentrations of (A) naringenin or (B) ABT-737, or (C) with $40 \mu \mathrm{M}$ naringenin and/or $5 \mu \mathrm{M}$ ABT-737, for $48 \mathrm{~h}$. Cell viability was determined using the cell counting kit- $8 .{ }^{* *} \mathrm{P}<0.01$; ${ }^{* * *} \mathrm{P}<0.001$.

Statistical analysis. Statistically significant differences between the treatment groups were determined using the Student's t-test or one-way analysis of variance, and were indicated by $\mathrm{P}<0.05$. Standard deviations in the data are demonstrated by a bar in the histogram.

\section{Results}

Naringenin and ABT-737 inhibit SGC7901 cell viability. Since naringenin and ABT-737 have anticancer activities $(7,8,13)$, the present study evaluated their effect on SGC7901 cell growth using the CCK-8 assay. Treatment with naringenin or ABT-737 was found to inhibit SGC7901 cell growth in a dose-dependent manner (Fig. 1A and B). Subsequently, the combined effects of naringenin and ABT-737 on cell growth were determined; cell viability was significantly decreased upon treatment of the SGC7901 cells with the two drugs, as compared with that observed with either drug alone $(\mathrm{P}<0.001$; Fig. 1C). These results indicated that naringenin was able 
A
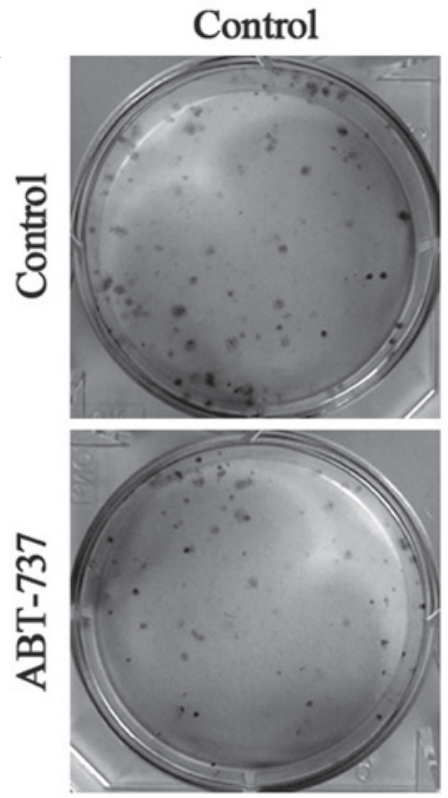

Naringenin
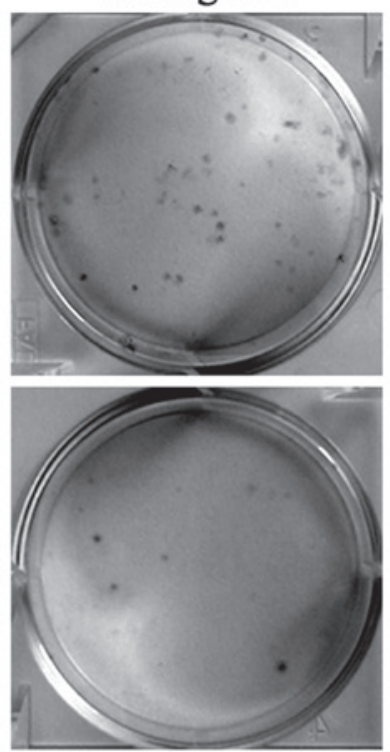

B

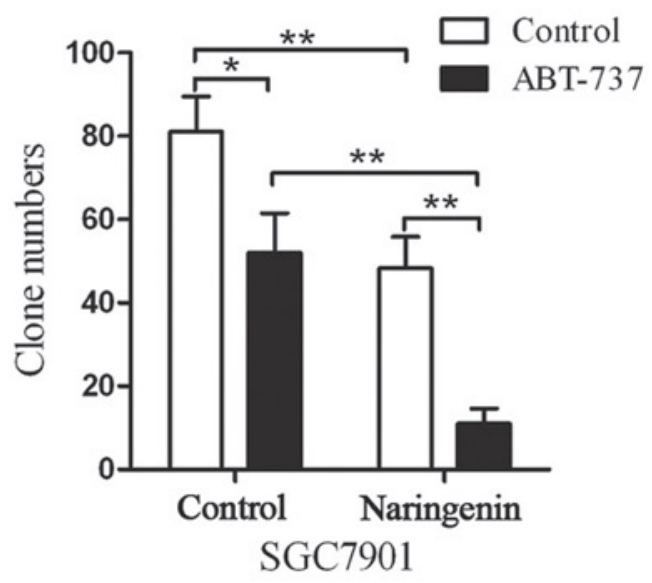

Figure 2. Naringenin and ABT-737 inhibit colony formation of SGC7901 cells. (A) Representative images of SGC7901 cells treated with $40 \mu \mathrm{M}$ naringenin and/or $5 \mu$ M ABT-737 for 6 days and stained with crystal violet. (B) Number of colonies counted and presented as a histogram. ${ }^{*} \mathrm{P}<0.05,{ }^{* * *} \mathrm{P}<0.01$.
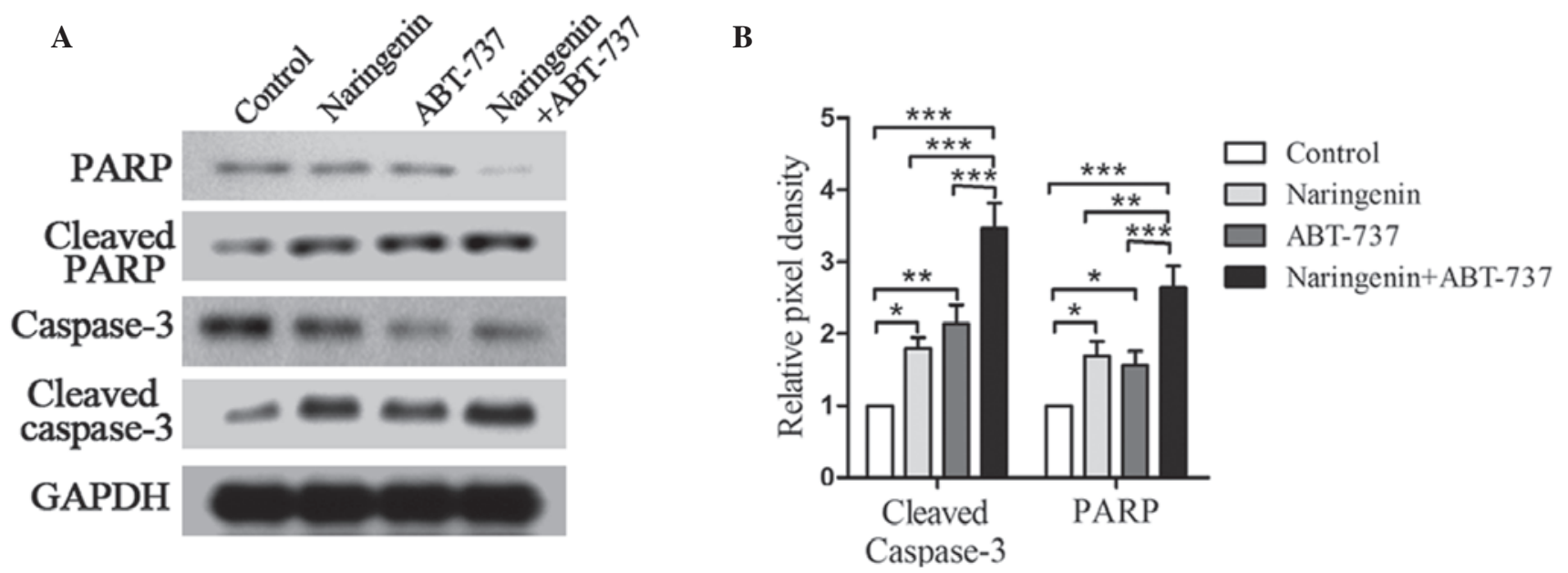

Figure 3. Naringenin and ABT-737 induced cleavage of capase-3 and PARP. (A) Western blots of SG7901 cells treated $40 \mu \mathrm{M}$ naringenin and/or $5 \mu \mathrm{M}$ ABT-737 for $48 \mathrm{~h}$, detecting the protein expression levels of caspase-3 and PARP. GAPDH was included as a loading control. (B) Relative pixel density of cleaved caspase-3 and PARP was measured and normalized against GAPDH. PARP, poly ADP-ribose polymerase; GAPDH, glyceraldehyde-3-phosphate dehydrogenase. ${ }^{*} \mathrm{P}<0.05,{ }^{* *} \mathrm{P}<0.01$ and ${ }^{* * *} \mathrm{P}<0.001$.

to inhibit gastric cancer cell growth, and that the inhibition induced by naringenin was enhanced upon addition of ABT-737.

Naringenin and ABT-737 inhibit SGC7901 cell colony formation. Naringenin and ABT-737 were found to inhibit SGC7901 cell growth; thus, this inhibition was investigated using a colony formation assay. A low density of SGC7901 cells were seeded and treated with naringenin and/or ABT-737 for 6 days (Fig. 2A). Treatment with naringenin or ABT-737 alone decreased the clone numbers by $\sim 40 \%$, while a combination of the two drugs induced a $90 \%$ decrease in cell colonies, as compared with the control group (Fig. 2B). Therefore, SGC7901 cell colony formation was inhibited by naringenin, ABT-737 or a combination of the two.
Naringenin and ABT-737 induce more cleaved caspase-3 and PARP. Naringenin and ABT-737 have been shown to induce apoptosis in numerous types of cancer cells (18-21); therefore, the apoptotic effects of these two drugs on SGC7901 cells was investigated. The expression levels of the apoptosis-associated proteins caspase- 3 and PARP were detected in SGC7901 cells using western blot analysis (Fig. 3). Treatment with naringenin or ABT-737 alone significantly increased the expression levels of cleaved caspase- 3 and PARP ( $<<0.05$; Fig. $3 \mathrm{~A}$ and $\mathrm{B})$, as compared with the control cells. However, the expression levels of cleaved caspase-3 and PARP were more significantly increased following the combination treatment $(\mathrm{P}<0.01$; Fig. 3A and B); as compared with either treatment alone. These results suggested an enhancement in the apoptosis of SGC7901 cells following treatment with naringenin and ABT737. 

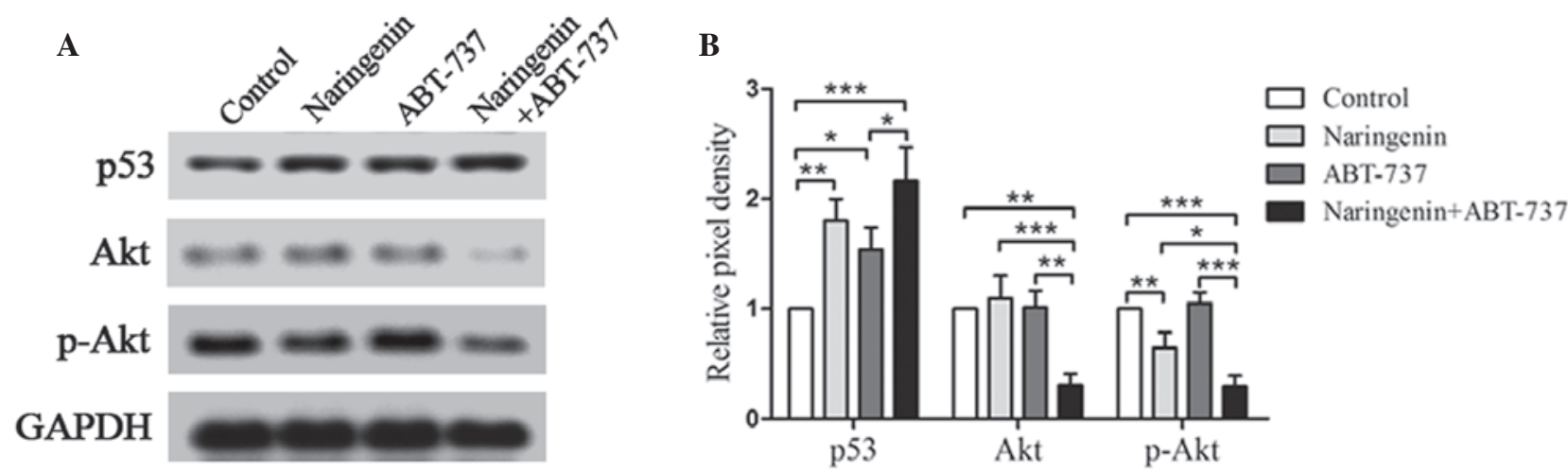

Figure 4. Naringenin and ABT-737 inhibited Akt and increased p53 expression. (A) Western blots of SGC7901 cells treated $40 \mu \mathrm{M}$ naringenin and/or $5 \mu \mathrm{M}$ ABT-737 for $48 \mathrm{~h}$, showing the expression levels of p53, Akt and p-Akt. GAPDH was included as a loading control. (B) Relative pixel density of p53, Akt and p-Akt normalized against GAPDH and presented as a histogram. Akt, protein kinase B; p-Akt, phospho-Akt; GAPDH, glyceraldehyde-3-phosphate dehydrogenase. ${ }^{*} \mathrm{P}<0.05,{ }^{* *} \mathrm{P}<0.01$ and ${ }^{* * *} \mathrm{P}<0.001$.

Naringenin and ABT-737 increase 53 expression and reduce Akt activation. Naringenin is able to induce cell apoptosis via multiple pathways $(9,22,23)$. In the present study, the pathways involved in naringenin and ABT-737-induced cell apoptosis, including the p53 and Akt pathways that are important in cell apoptosis and survival $(24,25)$, were investigated (Fig. 4). The results indicated that naringenin inhibited the phosphorylation of Akt, whereas ABT-737 did not have the same effect. The expression levels and rate of phosphorylation of Akt in SGC7901 cells were significantly reduced upon treatment with a combination of naringenin and ABT-737 $(\mathrm{P}<0.05)$. Furthermore, naringenin and/or ABT-737 increased the expression levels of p53 protein. These results suggested that the p53 and Akt pathways are involved in the cell apoptosis induced by the combination of naringenin and ABT-737 treatment of SGC7901 cells.

\section{Discussion}

The present study demonstrated anticancer activities for naringenin and ABT-737, and that naringenin, in combination with ABT-737, was able to inhibit gastric cancer cell growth and colony formation. In addition, naringenin and ABT-737 induced the cleavage of caspase- 3 and PARP, which resulted in apoptosis. Furthermore, the p53 and Akt pathways were confirmed to be involved in the anticancer effects induced by the combination of these two drugs.

Naringenin is extracted from citrus fruit and previous studies have demonstrated its antimutagenic and anticarcinogenic activities in various cancer types $(26,27)$. Ekambaram et al $(7,28)$ reported the modulatory and anticancer effects of naringenin on gastric carcinogenesis induced by N-methyl-N'-nitro-N-nitrosoguanidine and saturated sodium chloride in rats. However, the biological activity of naringenin on human gastric cancer cells has not been previously investigated. The results of the present study demonstrated that naringenin was able to induce the inhibition of gastric cancer cell growth and colony formation. This inhibitory effect may be caused by naringenin-induced apoptosis through the activation of caspase-3. Naringenin also increased the expression levels of p53 and downregulated Akt activation in the SGC7901 cells.
Previous studies have shown that naringenin induced apoptosis in acute myeloid leukemia cells through the downregulation of Akt and caspase-3 activation (19). A recent study also demonstrated that naringenin was able to inhibit bladder cancer cell migration through the downregulation of the Akt and matrix metalloproteinase-2 pathways (8). These observations are consistent with the findings of the present study.

Drug resistance is a feature of gastric cancer that is associated with poor prognosis. The anti-apoptotic protein Bcl-2 is overexpressed in various types of cancer cells and is associated with drug resistance (12). Novel drugs targeting Bcl-2, including ABT-737, have been developed to overcome the drug resistance and induce the apoptosis of cancer cells. In the present study, the inhibitory effects of ABT-737 on cell growth and colony formation were confirmed in gastric cancer cells. Similar to the results of the present study, a previous study also reported the pro-apoptotic role of ABT-737 in gastric cancer cells (17). In addition, ABT-737 in combination with naringenin was found to further inhibit SGC7901 cell growth and colony formation in the current study. Our results also showed that naringenin and ABT-737 were able to further increase cleavage of caspse- 3 and PARP, leading to the inhibition of cell growth and survival. Furthermore, these two drugs increased p53 expression, which has a positive role in cell apoptosis. The Akt pathway, which is a pro-survival pathway, was inhibited by naringenin alone or in combination with ABT-737.

In conclusion, naringenin and ABT-737 were found to exhibit an anticancer activity in gastric cancer cells. Naringenin alone or in combination with ABT-737 was able to induce the inhibition of gastric cancer cell growth and colony formation. In addition, naringenin and ABT-737 induced the activation of caspase- 3 and cleavage of PARP, leading to cell apoptosis. Finally, the Akt and p53 pathways were found to be involved in the combination effect of naringenin and ABT-737 in gastric cancer cells.

\section{References}

1. Li Y, Yan PW, Huang XE and Li CG: MDR1 gene C3435T polymorphism is associated with clinical outcomes in gastric cancer patients treated with postoperative adjuvant chemotherapy. Asian Pac J Cancer Prev 12: 2405-2409, 2011. 
2. Ferro A, Peleteiro B, Malvezzi M, Bosetti C, Bertuccio P, Levi F, Negri E, La Vecchia C and Lunet N: Worldwide trends in gastric cancer mortality (1980-2011), with predictions to 2015, and incidence by subtype. Eur J Cancer 50: 1330-1344, 2014.

3. American Cancer Society: Survival rates for stomach cancer, by stage. www.cancer.org/cancer/stomachcancer/detailedguide/ stomach-cancer-survival-rates. Accessed June 24, 2014.

4. Shan YS, Hsu HP, Lai MD, Yen MC, Luo YP and Chen YL: Increased expression of argininosuccinate synthetase protein predicts poor prognosis in human gastric cancer. Oncology Rep 33: 49-57, 2015.

5. Yuan J, Li W, Tian Y and Wang X: Anti-proliferative effect of Flos Albiziae flavonoids on the human gastric cancer SGC-7901 cell line. Exp Ther Med 5: 51-56, 2013.

6. Romagnolo DF and Selmin OI: Flavonoids and cancer prevention: A review of the evidence. J Nutr Gerontol Geriatr 31: 206-238, 2012.

7. Ekambaram G, Rajendran P, Magesh V and Sakthisekaran D: Naringenin reduces tumor size and weight lost in $\mathrm{N}$-methyl-N'-nitro-N-nitrosoguanidine-induced gastric carcinogenesis in rats. Nutr Res 28: 106-112, 2008.

8. Liao AC, Kuo CC, Huang YC, Yeh CW, Hseu YC, Liu JY and Hsu LS: Naringenin inhibits migration of bladder cancer cells through downregulation of AKT and MMP2. Mol Med Rep 10: 1531-1536, 2014

9. Hatkevich T, Ramos J, Santos-Sanchez I and Patel YM: A naringenin-tamoxifen combination impairs cell proliferation and survival of MCF-7 breast cancer cells. Exp Cell Res 327: 331-339, 2014.

10. Ahamad MS, Siddiqui S, Jafri A, Ahmad S, Afzal M and Arshad M: Induction of apoptosis and antiproliferative activity of naringenin in human epidermoid carcinoma cell through ROS generation and cell cycle arrest. PloS one 9: e110003, 2014.

11. Arul D and Subramanian P: Naringenin (citrus flavonone) induces growth inhibition, cell cycle arrest and apoptosis in human hepatocellular carcinoma cells. Pathol Oncol Res 19: 763-770, 2013

12. Kang MH and Reynolds CP: Bcl-2 inhibitors: Targeting mitochondrial apoptotic pathways in cancer therapy. Clin Cancer Res 15: 1126-1132, 2009.

13. Tahir SK, Yang X, Anderson MG, Morgan-Lappe SE, Sarthy AV Chen J, Warner RB, Ng SC, Fesik SW, Elmore SW, et al: Influence of Bcl-2 family members on the cellular response of small-cell lung cancer cell lines to ABT-737. Cancer Res 67: 1176-1183, 2007.

14. Konopleva M, Contractor R, Tsao T, Samudio I, Ruvolo PP, Kitada S, Deng X, Zhai D, Shi YX, Sneed T, et al: Mechanisms of apoptosis sensitivity and resistance to the BH3 mimetic ABT-737 in acute myeloid leukemia. Cancer Cell 10: 375-388, 2006 .
15. Bodet L, Gomez-Bougie P, Touzeau C, Dousset C, Descamps G, Maïga S, Avet-Loiseau H, Bataille R, Moreau P, Le Gouill S, et al: ABT-737 is highly effective against molecular subgroups of multiple myeloma. Blood 118: 3901-3910, 2011.

16. van Delft MF, Wei AH, Mason KD, Vandenberg CJ, Chen L, Czabotar PE, Willis SN, Scott CL, Day CL, Cory S, et al: The BH3 mimetic ABT-737 targets selective Bcl-2 proteins and efficiently induces apoptosis via Bak/Bax if Mcl-1 is neutralized. Cancer cell 10: 389-399, 2006.

17. Sun XP, Zhang X, He C, Qiao H, Jiang X, Jiang H and Sun X: ABT-737 synergizes with arsenic trioxide to induce apoptosis of gastric carcinoma cells in vitro and in vivo. J Int Med Res 40: 1251-1264, 2012.

18. Li RF, Feng YQ, Chen JH, Ge LT, Xiao SY and Zuo XL: Naringenin suppresses K562 human leukemia cell proliferation and ameliorates Adriamycin-induced oxidative damage in polymorphonuclear leukocytes. Exp Ther Med 9: 697-706, 2015.

19. Park JH, Jin CY, Lee BK, Kim GY, Choi YH and Jeong YK: Naringenin induces apoptosis through downregulation of Akt and caspase- 3 activation in human leukemia THP-1 cells. Food Chem Toxicol 46: 3684-3690, 2008.

20. Parrondo R, de Las Pozas A, Reiner T and Perez-Stable C: ABT-737, a small molecule Bcl-2/Bcl-xL antagonist, increases antimitotic-mediated apoptosis in human prostate cancer cells PeerJ 1: e144, 2013.

21. Cristofanon S and Fulda S: ABT-737 promotes tBid mitochondrial accumulation to enhance TRAIL-induced apoptosis in glioblastoma cells. Cell Death Dis 3: e432, 2012.

22. Yu DH, Ma CH, Yue ZQ, Yao X and Mao CM: Protective effect of naringenin against lipopolysaccharide-induced injury in normal human bronchial epithelium via suppression of MAPK signaling. Inflammation 38: 195-204, 2015.

23. Kapoor R, Rizvi F and Kakkar P: Naringenin prevents high glucose-induced mitochondria-mediated apoptosis involving AIF, Endo-G and caspases. Apoptosis 18: 9-27, 2013.

24. Kruiswijk F, Labuschagne CF and Vousden KH: p53 in survival, death and metabolic health: A lifeguard with a licence to kill. Nat Rev Mol Cell Biol 16: 393-405, 2015.

25. Warfel NA and Kraft AS: PIM kinase (and Akt) biology and signaling in tumors. Pharmacol Ther 151: 41-49, 2015.

26. Alam MA, Subhan N, Rahman MM, Uddin SJ, Reza HM and Sarker SD: Effect of citrus flavonoids, naringin and naringenin, on metabolic syndrome and their mechanisms of action. Adv Nutr 5: 404-417, 2014

27. Sabarinathan D, Mahalakshmi P and Vanisree AJ: Naringenin promote apoptosis in cerebrally implanted C6 glioma cells. Mol Cell Biochem 345: 215-222, 2010.

28. Ekambaram G, Rajendran P, Devaraja R, Muthuvel R and Sakthisekaran D: Impact of naringenin on glycoprotein levels in N-methyl-N'-nitro-N-nitrosoguanidine-induced gastric carcinogenesis in rats. Anticancer Drugs 19: 885-890, 2008 . 